TRANSACTIONS OF THE

AMERICAN MATHEMATICAL SOCIETY

Volume 364, Number 2, February 2012, Pages 703-719

S 0002-9947(2011)05322-5

Article electronically published on September 13, 2011

\title{
A LIMITING FREE BOUNDARY PROBLEM RULED BY ARONSSON'S EQUATION
}

\author{
JULIO D. ROSSI AND EDUARDO V. TEIXEIRA
}

\begin{abstract}
We study the behavior of a $p$-Dirichlet optimal design problem with volume constraint for $p$ large. As the limit of $p$ goes to infinity, we find a limiting free boundary problem governed by the infinity-Laplacian operator. We find a necessary and sufficient condition for uniqueness of the limiting problem and, under such a condition, we determine precisely the optimal configuration for the limiting problem. Finally, we establish convergence results for the free boundaries.
\end{abstract}

\section{INTRODUCTION}

Let $\Omega$ be a smooth bounded domain in the Euclidean space $\mathbb{R}^{n}$ and $\alpha$ a fixed positive number less than the Lebesgue measure of $\Omega$. An optimal design problem with volume constraint can be generally written as

$$
\operatorname{Min}\left\{\mathfrak{J}(\mathcal{O}) \mid \mathcal{O} \subset \Omega \quad \text { and } \quad \mathcal{L}^{n}(\mathcal{O}) \leq \alpha\right\} .
$$

For most applications, $\mathfrak{J}(\mathcal{O})$ has an integral representation involving functions which are linked to the competing configuration $\mathcal{O}$ by a prescribed partial differential equation (PDE).

The modern history of this line of research probably starts at the pioneering work of N. Aguilera, W. Alt and L. Caffarelli 2]. In that paper, the authors address the question of minimizing the Dirichlet integral when prescribed the volume of the zero set. C. Lederman in [17] establishes similar results for the nonhomogeneous minimization problem, $\int|D u|^{2}-g u$. N. Aguilera, L. Caffarelli and J. Spruck [4] considered the minimization problem (1.1) for $\mathfrak{J}(\mathcal{O})=\int_{\Omega} \Delta u d X$, where $u$ is the harmonic function in $\mathcal{O}$, taking a prescribed boundary data $\varphi$ on $\partial \Omega$ and zero on $\partial \mathcal{O}$. This is a model for an optimal shape problem in heat conduction theory with nonconstant temperature distribution. Nonlinear optimal design problems with nonconstant temperature distribution were treated in 22. The common feature of the aforementioned works is that all of them are governed by the Laplacian operator. Their fine analyses rely on the revolutionary work of W. Alt and L. Caffarelli [3].

Just recently the study of optimal design problems ruled by degenerate quasilinear operators was successfully developed. This theory is the starting point for the main goal of the present work, which we now describe. Let us consider the problem of minimizing the $p$-Dirichlet integral with a given positive boundary data

Received by the editors March 24, 2009 and, in revised form, February 9, 2010.

2010 Mathematics Subject Classification. Primary 35R35, 35J70, 62K05, 49L25.

Key words and phrases. Optimal design, free boundary problems, infinite Laplacian. 
$f$ and with the maximum volume of the support prescribed. More precisely, let us consider the following free boundary optimization problem:

$\left(\mathfrak{P}_{p}\right) \min \left\{\int_{\Omega}|\nabla u(X)|^{p} d X \mid u \in W^{1, p}(\Omega), u=f\right.$ on $\left.\partial \Omega, \mathcal{L}^{n}(\{u>0\}) \leq \alpha\right\}$.

Existence of a minimizer as well as smoothness properties of its free boundary have been established in [10] and [21]. Further generalizations are addressed in 25] and 26]. In the present paper, we are interested in the asymptotic behavior, as $p$ goes to infinity, of optimal shapes to problem $\left(\mathfrak{P}_{p}\right)$. Analytical and geometric features of a limiting free boundary reveals asymptotic information upon the optimal design problem $\left(\mathfrak{P}_{p}\right)$. Driven by classical considerations, we are led to consider the following limiting problem:

$\left(\mathfrak{P}_{\infty}\right) \quad \min \left\{\operatorname{Lip}(u) \mid u \in W^{1, \infty}(\Omega), u=f\right.$, on $\left.\partial \Omega, \mathcal{L}^{n}(\{u>0\}) \leq \alpha\right\}$,

where $\operatorname{Lip}(u)$ is the Lipschitz constant of $u$ :

$$
\operatorname{Lip}(u)=\sup _{x, y} \frac{|u(x)-u(y)|}{|x-y|} .
$$

Our first concern is to prove that any sequence of minimizers $u_{p}$ to problem $\left(\mathfrak{P}_{p}\right)$ converges (up to a subsequence) to a solution, $u_{\infty}$, of the limiting problem $\left(\mathfrak{P}_{\infty}\right)$. In addition, we are interested in finding the partial differential equation (PDE) $u_{\infty}$ satisfies in its set of positivity. In this direction and enforcing the fact that $u_{\infty}$ is an extremal for the Lipschitz minimization problem, we show that $u_{\infty}$ is indeed an absolute minimizer for the Lipschitz constant within its set of positivity, $\Omega_{\infty}:=\left\{u_{\infty}>0\right\}$. That is, it minimizes the Lipschitz constant in every subdomain of $\Omega_{\infty}$ when testing against functions with the same boundary data; see [5]. Hence, it is an $\infty$-harmonic function in its positivity set. This information is the content of the first theorem in this paper that we state now.

Theorem 1. Let $u_{p}$ be a minimizer of $\left|\mathfrak{P}_{p}\right|$. Then, up to a subsequence,

$$
u_{p} \rightarrow u_{\infty}, \quad \text { as } p \rightarrow \infty,
$$

uniformly in $\bar{\Omega}$ and weakly in every $W^{1, q}(\Omega)$ for $1<q<\infty$, where $v_{\infty}$ is a minimizer of $\left(\mathfrak{P}_{\infty}\right)$. The limiting function $u_{\infty}$ satisfies the PDE, $\Delta_{\infty} u_{\infty}=0$, in $\left\{u_{\infty}>0\right\}$ in the viscosity sense. Here $\Delta_{\infty} u:=D u D^{2} u(D u)^{t}$ is the infinityLaplacian.

It is known that under the assumptions $\Omega$ convex and $f \equiv$ const., one can prove uniqueness for problem $(\mathfrak{P} p), 23$. (see also 1, 12, 13, 14, 16] for related Bernoullitype problems). However, uniqueness is not expected in general for problem $\left(\mathfrak{P}_{p}\right)$. Surprisingly enough, under a mild compatibility condition upon $\operatorname{Lip}(f), \Omega$, and $\alpha$ which does not involve any convexity assumption on $\Omega$, we prove uniqueness for the limiting problem $\left(\mathfrak{P}_{\infty}\right)$. In particular, in this case any sequence of solutions to problem $\left[\mathfrak{P}_{p}\right.$ converges to the same optimal limiting configuration. Such a result can be read as an asymptotic uniqueness phenomenon for problem $\left(\mathfrak{P}_{p}\right.$. In addition, we have precisely found the optimal shape for the limiting problem $\left(\mathfrak{P}_{\infty}\right)$; that is, it reveals where and how optimal configurations $\Omega_{p}:=\left\{u_{p}>0\right\}$ stabilize (see also Remark 2). 
More precisely, for our next theorem we shall work under the following geometric compatibility condition:

$$
\mathcal{L}^{n}\left(\bigcup_{y \in \partial \Omega} B_{\frac{f(y)}{\operatorname{Lip}(f)}}(y) \cap \Omega\right) \geq \alpha .
$$

It is understood that if $f$ is constant, then $(\underline{\mathrm{H}})$ is automatically satisfied.

Theorem 2. Assume $(\underline{\mathrm{H}})$, and let $\lambda^{\star}$ be the unique positive real number such that the domain

$$
\Omega^{\star}:=\bigcup_{x \in \partial \Omega} B_{\frac{f(x)}{\lambda \star}}(x) \cap \Omega
$$

has Lebesgue measure precisely $\alpha$. Then the function $u_{\infty}$, defined as

$$
\left\{\begin{array}{rlrl}
\Delta_{\infty} u_{\infty} & =0 & & \text { in } \Omega^{\star}, \\
u_{\infty}=f & & \text { on } \partial \Omega, \\
u_{\infty} & =0 & & \text { on } \partial \Omega^{\star} \cap \Omega
\end{array}\right.
$$

is the unique minimizer for problem $\left(\mathfrak{P}_{\infty}\right)$. Hence, if $u_{p}$ is a minimizer of $\left(\mathfrak{P}_{p}\right)$, then the whole sequence $u_{p}$ converges, $u_{p} \rightarrow u_{\infty}$, uniformly in $\bar{\Omega}$ and weakly in every $W^{1, q}(\Omega)$ for $1<q<\infty$. In addition, $u_{\infty}$ is given by the formula,

$$
u_{\infty}=\max _{y \in \partial \Omega}\left(f(y)-\lambda^{\star}|x-y|\right)_{+} \cdot
$$

Remark 1. As mentioned above, Theorem 2 applies in particular to an important physical situation, namely heat conduction problems with evenly heated domains, i.e., $f \equiv T$ (constant).

Remark 2. From the applied point of view, Theorem 2 provides a rigorous mathematical proof for the empirical, and widely employed, intuition that says that the configuration $\Omega^{\star}$ should be approximately an optimal way of insulating a given body $\Omega$ with temperature distribution $f$.

Remark 3. The fact that the equation that rules the limit configuration is Aronsson's equation $-\Delta_{\infty} u=0$ is not surprising. Infinity harmonic functions (solutions to $-\Delta_{\infty} u=0$ ) appear naturally as limits of $p$-harmonic functions (solutions to $\left.\Delta_{p} u=\operatorname{div}\left(|\nabla u|^{p-2} \nabla u\right)=0\right)$, [6], and have applications to optimal transport problems, [9], 11], image processing, etc.; see the survey [5].

In view of Theorem 2 it becomes natural to inquire what happens if condition $(\underline{\mathrm{H}})$ is violated. In this direction, we show that $(\underline{\mathrm{H}})$ is a necessary and sufficient condition for uniqueness to problem $\left(\mathfrak{P}_{\infty}\right)$. Indeed, if $(\underline{\mathbb{H}})$ does not hold, we manage to find multiple solutions for problem $\left(\overline{\mathfrak{P}_{\infty}}\right)$. Nevertheless, we could prove the existence of a minimal one.

Theorem 3. Assume that $(\mathrm{H})$ does not hold. Then there exists infinitely many minimizers for the limit problem $\left(\mathfrak{P}_{\infty}\right)$. The function

$$
u_{\infty}(x)=\max _{y \in \partial \Omega}(f(y)-\operatorname{Lip}(f)|x-y|)_{+}
$$

is a minimizer with the measure of its positivity set

$$
\left\{u_{\infty}>0\right\}=\bigcup_{x \in \partial \Omega} B_{\frac{f(x)}{\operatorname{Lip}(f)}}(x) \cap \Omega
$$

strictly less than $\alpha$. Moreover, $u_{\infty}$ is the minimal solution, in the sense that any minimizer $v_{\infty}$ verifies $v_{\infty}(x) \geq u_{\infty}(x)$. 
Remark 4. Note that the support of the minimal minimizer for problem $\left[\mathfrak{P}_{\infty} \mid\right.$ is given by the set $\Omega^{\star}:=\bigcup_{x \in \partial \Omega} B_{\frac{f(x)}{\operatorname{Lip}(f)}}(x) \cap \Omega$.

Finally, we study geometric properties of the limiting free boundary, $\partial\left\{u_{\infty}>0\right\}$, as well as convergence issues of the free boundaries $\partial\left\{u_{p}>0\right\}$. The next theorem we state shows that the the limiting free boundary enjoys the appropriate geometric features suitable for the study of its geometric measure properties.

Theorem 4. Let $u_{p}$ be extremals to problem $\sqrt{\mathfrak{P}_{p}}$, and assume that a subsequence (still labelled as $u_{p}$ ) is such that $u_{p} \rightarrow u_{\infty}$ uniformly in $\bar{\Omega}$ and weakly in every $W^{1, q}(\Omega)$ for $1<q<\infty$. Then $u_{\infty}$ is uniformly Lipschitz continuous in $\Omega$, grows linearly away from the free boundary, and is strongly nondegenerate. That is, for a constant $\gamma>0$,

$$
u_{\infty}(x) \geq \gamma \operatorname{dist}\left(x, \partial\left\{u_{x}>0\right\}\right), \quad \forall x \in \Omega_{\infty}:=\left\{u_{\infty}>0\right\},
$$

and for any fixed free boundary point $x_{0} \in \partial\left\{u_{\infty}>0\right\}$ there holds

$$
\sup _{B_{r}\left(x_{0}\right)} u_{\infty} \geq \gamma r .
$$

The strategy for showing Theorem 4 is to revisit the $p$-Dirichlet optimization problem $\left(\mathfrak{P}_{p}\right)$ and verify that these properties hold uniformly in $p$. As a by-product of this analysis, we obtain convergence of the free boundaries $\partial\left\{u_{p}>0\right\}$ in the Hausdorff metric.

Theorem 5. Let $u_{p}$ be a sequence of minimizers for problem $\left(\mathfrak{P}_{p}\right)$, and assume that a subsequence of $u_{p}$ is such that $u_{p} \rightarrow u_{\infty}$ uniformly in $\bar{\Omega}$ and weakly in every $W^{1, q}(\Omega)$ for $1<q<\infty, u_{\infty}$ being a solution to $\left[\mathfrak{P}_{\infty}\right.$. Then

$$
\partial\left\{u_{p}>0\right\} \longrightarrow \partial\left\{u_{\infty}>0\right\}, \quad \text { as } p \rightarrow \infty,
$$

in the Hausdorff distance.

The variational optimization problem $\left(\mathfrak{P} p_{p}\right)$ relates, to some extent, to Bernoullitype problems governed by the $p$-Laplacian operator. This is done through a constant free boundary condition proven to hold for minimizers of problem $(\mathfrak{P p})$. Indeed, it has been shown (see [10, 21]) that $\left|\nabla u_{p}\right|=\lambda_{u_{p}}$ for a positive constant $\lambda_{u_{p}}$ along its free boundary $\partial\left\{u_{p}>0\right\}$. This is the so-called free boundary condition for the optimization problem $\left[\mathfrak{P} p_{p} \mid\right.$ - a key piece of information when studying geometric measure as well as smoothness properties of the free boundary. In this direction we have proven the following convergence of free boundary conditions.

Theorem 6. Let $u_{p}$ be a sequence of minimizers for problem $\left(\mathfrak{P}_{p}\right)$ and $\left|\nabla u_{p}\right|=\lambda_{p}$ along $\partial\left\{u_{p}>0\right\}$. Denote $\Omega_{\infty}:=\left\{u_{\infty}>0\right\}$. Then, up to a subsequence, $\left(u_{p}, \lambda_{p}\right) \rightarrow$ $\left(u_{\infty}, \lambda_{\infty}\right)$, with $0<\lambda_{\infty}<\infty$ and

$$
\lim _{\substack{x \rightarrow \partial \Omega_{\infty} \\ x \in \Omega_{\infty}}} \frac{u_{\infty}(x)}{\operatorname{dist}\left(x, \partial \Omega_{\infty}\right)}=\lambda_{\infty} .
$$

When $\Omega$ is convex and $f$ is constant, Theorem [ 6 can be seen in connection to the results of J. J. Manfredi, A. Petrosyan and H. Shahgholian 18, who study convergence issues, as $p \rightarrow \infty$, for Bernoulli-type problems.

The rest of the paper is organized as follows: In the next section we prove Theorem 1. In Section 3 we study the limit problem under condition $(\mathbb{H})$ and in 
Section 4 we deal with the complementary case. Finally, in Section 5 we include some uniform bounds for the sequence $u_{p}$ (showing uniform nondegeneracy of the free boundary), and we study the convergence of the free boundaries.

\section{Proof of Theorem 1}

In this section we prove Theorem 1. The key to the proof is to find bounds for the energy $\left(\int_{\Omega}\left|\nabla u_{p}\right|^{p}\right)^{1 / p}$ of a minimizer that are independent of $p$.

Proof of Theorem 1. Let us fix hereafter a Lipschitz extension of $f$, which we will denote by $v$, among functions in the set

$$
K_{\infty}=\left\{\varphi \in W^{1, \infty}(\Omega) \mid \varphi=f, \text { on } \partial \Omega,|\{\varphi>0\}|=\alpha\right\} .
$$

Clearly, since $\Omega$ is bounded, $v$ competes in the minimization problem $\left[\mathfrak{P} p_{p}\right]$. Thus, using $v$ as a test function in problem $\left(\overline{\mathfrak{P}_{p}}\right)$, we obtain

$$
\left(\int_{\Omega}\left|\nabla u_{p}\right|^{p}\right)^{1 / p} \leq\left(\int_{\Omega}|\nabla v|^{p}\right)^{1 / p} \leq \operatorname{Lip}(v)|\Omega|^{1 / p} \leq C,
$$

where $C$ is a constant independent of $p$. With an exponent $q<\infty$ fixed, we obtain

$$
\left(\int_{\Omega}\left|\nabla u_{p}\right|^{q}\right)^{1 / q} \leq\left(\int_{\Omega}\left|\nabla u_{p}\right|^{p}\right)^{1 / p}|\Omega|^{p /(q(p-q))} \leq \operatorname{Lip}(v)|\Omega|^{1 / p+p /(q(p-q))} \leq C .
$$

Therefore, the sequence $u_{p}$ is uniformly bounded in $W^{1, q}(\Omega)$, and its weak limit as $p \rightarrow \infty$ (let us call it $u_{\infty}$ ) verifies

$$
\left(\int_{\Omega}\left|\nabla u_{\infty}\right|^{q}\right)^{1 / q} \leq \operatorname{Lip}(v)|\Omega|^{1 / q} \leq C .
$$

Taking $q \rightarrow \infty$ and performing a diagonal argument, we obtain a subsequence (that will still be named as $u_{p}$ ) that converges weakly in every $W^{1, q}(\Omega), 1<q<\infty$ to a limit $u_{\infty} \in W^{1, \infty}(\Omega)$ such that

$$
\left\|\nabla u_{\infty}\right\|_{L^{\infty}(\Omega)} \leq \operatorname{Lip}(v) .
$$

Let us now turn our attention towards estimating the Lebesgue measure of $\left\{u_{\infty}>\right.$ $0\}$. Fix $\epsilon>0$. Thanks to uniform convergence, for $p$ large enough, there holds

$$
\left\{u_{\infty}>\epsilon\right\} \subset\left\{u_{p}>0\right\} .
$$

Hence we conclude that

$$
\mathcal{L}^{n}\left(\left\{u_{\infty}>0\right\}\right)=\lim _{\epsilon \rightarrow 0} \mathcal{L}^{n}\left(\left\{u_{\infty}>\epsilon\right\}\right) \leq \alpha .
$$

Therefore, we have proved that $u_{\infty}$ is an extremal for the limit problem $\left(\mathfrak{P}_{\infty}\right)$.

It remains to prove that $u_{\infty}$ is indeed $\infty$-harmonic in its set of positivity. Following [7, let us recall the definition of viscosity solution.

Definition 2.1. Consider the boundary value problem

$$
F\left(x, D u, D^{2} u\right)=0 \quad \text { in } \Omega .
$$

(1) A lower semicontinuous function $u$ is a viscosity supersolution if for every $\phi \in C^{2}(\bar{\Omega})$ such that $u-\phi$ has a strict minimum at the point $x_{0} \in \Omega$ with $u\left(x_{0}\right)=\phi\left(x_{0}\right)$ we have

$$
F\left(x_{0}, D \phi\left(x_{0}\right), D^{2} \phi\left(x_{0}\right)\right) \geq 0 .
$$


(2) An upper semicontinuous function $u$ is a subsolution if for every $\phi \in C^{2}(\bar{\Omega})$ such that $u-\phi$ has a strict maximum at the point $x_{0} \in \Omega$ with $u\left(x_{0}\right)=\phi\left(x_{0}\right)$ we have

$$
F\left(x_{0}, D \phi\left(x_{0}\right), D^{2} \phi\left(x_{0}\right)\right) \leq 0 .
$$

(3) Finally, $u$ is a viscosity solution if it is a super- and a subsolution.

If we have a weak $p$-harmonic function (in the sense of distribution) that is continuous, then it is a viscosity solution. This is the content of our next result.

Lemma 2.1. Let $u$ be a continuous weak solution of $\Delta_{p} u=0$ in some domain $\Omega$ for $p>2$. Then $u$ is a viscosity solution of

$$
-(p-2)|D u|^{p-4} \Delta_{\infty} u-|D u|^{p-2} \Delta u=0 \quad \text { in } \Omega .
$$

Proof. Let $x_{0} \in \Omega$ and a test function $\phi$ such that $u\left(x_{0}\right)=\phi\left(x_{0}\right)$ and $u-\phi$ has a strict minimum at $x_{0}$. We want to show that

$$
-(p-2)|D \phi|^{p-4} \Delta_{\infty} \phi\left(x_{0}\right)-|D \phi|^{p-2} \Delta \phi\left(x_{0}\right) \geq 0 .
$$

Assume that this is not the case. Then there exists a radius $r>0$ such that

$$
-(p-2)|D \phi|^{p-4} \Delta_{\infty} \phi(x)-|D \phi|^{p-2} \Delta \phi(x)<0,
$$

for every $x \in B\left(x_{0}, r\right)$. Set $m=\inf _{\left|x-x_{0}\right|=r}(u-\phi)(x)$, and let $\psi(x)=\phi(x)+m / 2$. This function $\psi$ verifies $\psi\left(x_{0}\right)>u\left(x_{0}\right)$ and

$$
-\operatorname{div}\left(|D \psi|^{p-2} D \psi\right)<0 .
$$

Multiplying by $(\psi-u)^{+}$extended by zero outside $B\left(x_{0}, r\right)$, we get

$$
\int_{\{\psi>u\}}|D \psi|^{p-2} D \psi D(\psi-u)<0 .
$$

Taking $(\psi-u)^{+}$as test function in the weak form of the equation, we get

$$
\int_{\{\psi>u\}}|D u|^{p-2} D u D(\psi-u)=0 .
$$

Hence,

$$
C(N, p) \int_{\{\psi>u\}}|D \psi-D u|^{p} \leq \int_{\{\psi>u\}}\left\langle|D \psi|^{p-2} D \psi-|D u|^{p-2} D u, D(\psi-u)\right\rangle<0,
$$

a contradiction. This proves that $u$ is a viscosity supersolution. The proof of the fact that $u$ is a viscosity subsolution runs as above, and we omit the details.

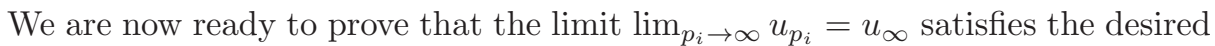
$\mathrm{PDE}$ in its set of positivity. In fact, let us check that $-\Delta_{\infty} u_{\infty}=0$ in the viscosity sense in the set $\left\{u_{\infty}>0\right\}$. Let us recall the standard proof. Let $\phi$ be a smooth test function such that $u_{\infty}-\phi$ has a strict maximum at $x_{0} \in\left\{u_{\infty}>0\right\}$. Since $u_{p_{i}}$ converges uniformly to $u_{\infty}$ we get that $u_{p_{i}}-\phi$ has a maximum at some point $x_{i} \in \Omega$ with $x_{i} \rightarrow x_{0}$ and moreover we have that $u_{p_{i}}>0$ in a whole fixed neighborhood of $x_{0}$ (and therefore $u_{p_{i}}\left(x_{i}\right)>0$ and every $u_{p_{i}}$ is $p$-harmonic there). Next, we use the fact that $u_{p_{i}}$ is a viscosity solution of $-\Delta_{p} u_{p}=0$ in the set $\left\{u_{p_{i}}>0\right\}$, and we obtain

$$
-\left(p_{i}-2\right)|D \phi|^{p_{i}-4} \Delta_{\infty} \phi\left(x_{i}\right)-|D \phi|^{p_{i}-2} \Delta \phi\left(x_{i}\right) \leq 0 .
$$


If $D \phi\left(x_{0}\right)=0$, we get $-\Delta_{\infty} \phi\left(x_{0}\right) \leq 0$. If this is not the case, we have that $D \phi\left(x_{i}\right) \neq 0$ for large $i$ and then

$$
-\Delta_{\infty} \phi\left(x_{i}\right) \leq \frac{1}{p_{i}-2}|D \phi|^{2} \Delta \phi\left(x_{i}\right) \rightarrow 0, \quad \text { as } i \rightarrow \infty .
$$

We conclude that

$$
-\Delta_{\infty} \phi\left(x_{0}\right) \leq 0 .
$$

That is, $u_{\infty}$ is a viscosity subsolution of $-\Delta_{\infty} u_{\infty}=0$.

A similar argument shows that $u_{\infty}$ is also a supersolution and therefore a solution of $-\Delta_{\infty} u_{\infty}=0$ in $\Omega$. The proof of Theorem 1 is completed.

\section{Proof of Theorem 2}

In this section we deal with the situation in which we have uniqueness for the limit problem. We will assume that condition (H) holds, that is,

$$
\mathcal{L}^{n}\left(\bigcup_{y \in \partial \Omega} B_{\frac{f(y)}{\operatorname{Lip}(f)}}(y) \cap \Omega\right) \geq \alpha .
$$

Note that with the notation of the statement of Theorem 2 this implies that

$$
\lambda^{\star} \geq \operatorname{Lip}(f) .
$$

This fact is crucial in the course of the next proof.

Proof of Theorem 2. Let $v_{\infty}$ be a minimizer for problem $\left(\overline{P_{\infty}}\right)$. Existence of such a minimizer is obtained by Theorem 1, Let us denote

$$
\Omega_{\infty}:=\left\{v_{\infty}>0\right\} \subset \Omega .
$$

For each free boundary point $y \in \partial \Omega_{\infty}$, let $x \in \partial \Omega$ be a point satisfying

$$
|x-y|=\operatorname{dist}(y, \partial \Omega) \text {. }
$$

Using the Lipschitz continuity of $v_{\infty}$, we obtain the following estimate:

$$
f(x) \leq \operatorname{Lip}\left(v_{\infty}\right)|x-y| .
$$

From (3.1), we immediately conclude that

$$
\bigcup_{x \in \partial \Omega} B_{\frac{f(x)}{\operatorname{Lip}\left(v_{\infty}\right)}}(x) \cap \Omega \subset \Omega_{\infty},
$$

which, in particular, implies

$$
\mathcal{L}^{n}\left(\bigcup_{x \in \partial \Omega} B_{\frac{f(x)}{\operatorname{Lip}\left(v_{\infty}\right)}}(x) \cap \Omega\right) \leq \alpha=\mathcal{L}^{n}\left(\bigcup_{x \in \partial \Omega} B_{\frac{f(x)}{\lambda x}}(x) \cap \Omega\right) .
$$

From above we obtain

$$
\lambda^{\star} \leq \operatorname{Lip}\left(v_{\infty}\right)
$$

On the other hand, let

$$
\Omega^{\star}:=\bigcup_{x \in \partial \Omega} B_{\frac{f(x)}{\lambda^{\star}}}(x) \cap \Omega
$$


Then, $u_{\infty}$, defined as the solution to

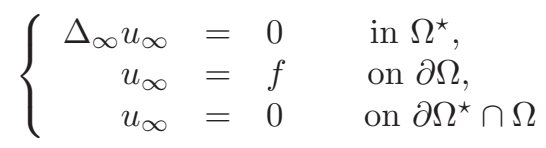

competes in the minimization problem $\left(\mathfrak{P}_{\infty}\right)$, thus

$$
\operatorname{Lip}\left(u_{\infty}\right) \geq \operatorname{Lip}\left(v_{\infty}\right) .
$$

In the sequel, we will use the fact that $u_{\infty}$ is the best Lipschitz extension of the boundary data $f$ on $\partial \Omega$ and 0 on $\partial \Omega^{\star} \cap \Omega$ together with the geometric compatibility condition (H) to bridge these inequalities. For that we consider the auxiliary barrier function

$$
\psi(x):=\max _{y \in \partial \Omega}\left(f(y)-\lambda^{\star}|x-y|\right)_{+} .
$$

We initially verify that

$$
\operatorname{Lip}(\psi)=\lambda^{\star} .
$$

To see this fact, let us first show that $\operatorname{Lip}(\psi) \leq \lambda^{\star}$. Let $x_{1}$ and $x_{2}$ be two points in $\Omega$. We assume $0<\psi\left(x_{1}\right)<\psi\left(x_{2}\right)$. Let $y_{1}$ and $y_{2}$ be such that

$$
\psi\left(x_{i}\right)=f\left(y_{i}\right)-\lambda^{\star}\left|x_{i}-y_{i}\right|, \quad \mathrm{i}=1,2 .
$$

From the definition of $\psi$, we know

$$
\psi\left(x_{1}\right) \geq f\left(y_{2}\right)-\lambda^{\star}\left|x_{1}-y_{2}\right| .
$$

We now estimate

$$
\begin{aligned}
0<\psi\left(x_{2}\right)-\psi\left(x_{1}\right) & \leq f\left(y_{2}\right)-\lambda^{\star}\left|x_{2}-y_{2}\right|-\left(f\left(y_{2}\right)-\lambda^{\star}\left|x_{1}-y_{2}\right|\right) \\
& \leq \lambda^{\star}\left(\left|x_{1}-y_{2}\right|-\left|x_{2}-y_{2}\right|\right) \\
& \leq \lambda^{\star}\left|x_{1}-x_{2}\right| .
\end{aligned}
$$

This shows that $\operatorname{Lip}(\psi) \leq \lambda^{\star}$. To see the reverse inequality, we argue as follows. Given $x_{1} \in \Omega$, let $y_{1}$ such that

$$
\psi\left(x_{1}\right)=f\left(y_{1}\right)-\lambda^{\star}\left|x_{1}-y_{1}\right| .
$$

Now, let $x_{2}=x_{1}+\gamma\left(x_{1}-y_{1}\right)$ and choose $\gamma$ small such that $x_{2} \in \Omega$. For this $x_{2}$ we also have that

and this implies

$$
\psi\left(x_{2}\right)=f\left(y_{1}\right)-\lambda^{\star}\left|x_{2}-y_{1}\right|,
$$

$$
\left|\psi\left(x_{1}\right)-\psi\left(x_{2}\right)\right|=\lambda^{\star}\left|x_{1}-x_{2}\right|,
$$

showing that $\operatorname{Lip}(\psi) \geq \lambda^{\star}$.

Our next step is to check that $\psi$ matches the desired boundary conditions. It is clear from its definition that

$$
\left.\psi\right|_{\partial \Omega^{\star}}=0
$$

Proving $\psi$ agrees with $f$ on $\partial \Omega$ is equivalent to showing that

$$
f(x)=\max _{y \in \partial \Omega}\left\{\left(f(y)-\lambda^{\star}|x-y|\right)_{+}\right\}, \quad \forall x \in \partial \Omega .
$$

Let us assume, for the sake of contradiction, that (3.7) does not hold. This will imply that there exist two points $x, y$ on $\partial \Omega$ with

$$
\lambda^{\star}|x-y|<f(y)-f(x) .
$$


That is,

$$
\lambda^{\star}<\operatorname{Lip}(f)=\sup _{x, y \in \partial \Omega}\left\{\frac{|f(x)-f(y)|}{|x-y|}\right\},
$$

which contradicts $(\mathbb{H})$.

As a remark, note that when we take two points $x, y \in \partial \Omega$, we get

$$
|\psi(x)-\psi(y)|=|f(x)-f(y)| \leq \operatorname{Lip}(f)|x-y| .
$$

Thus, $\operatorname{Lip}(\psi)=\max \left(\lambda^{\star}, \operatorname{Lip}(f)\right)=\lambda^{\star}$.

Once it is verified that $\psi$ has the same boundary condition as $u_{\infty}$, we know

$$
\operatorname{Lip}\left(u_{\infty}\right) \leq \operatorname{Lip}(\psi)=\lambda^{\star}
$$

Now let us show that $u_{\infty}$ coincides with the barrier

$$
\psi(x)=\max _{y \in \partial \Omega}\left(f(y)-\lambda^{\star}|x-y|\right)_{+} .
$$

We have that $u_{\infty}$ is a minimizer for the limit problem, hence we must have

$$
u_{\infty}(x) \geq \max _{y \in \partial \Omega}\left(f(y)-\lambda^{\star}|x-y|\right)_{+} \cdot
$$

In fact, if we assume that this is not the case, then there exists $x_{0}$ such that $u_{\infty}\left(x_{0}\right)<\psi\left(x_{0}\right)$. Now, considering quotients that involve $x_{0}$ and points on $\partial \Omega$, we can easily conclude that $\operatorname{Lip}\left(u_{\infty}\right)>\lambda^{\star}=\operatorname{Lip}(\psi)$, a contradiction since $\psi$ is a competitor in the limit problem.

Therefore, we obtain that both functions have the same positivity set (both sets have the same measure and one is included in the other).

Now, arguing as before, assume that there exists $x_{0}$ such that $u_{\infty}\left(x_{0}\right)>\psi\left(x_{0}\right)$. In this case, comparing quotients defining the Lipschitz constant with $x_{0}$ and points on the boundary of the positivity set, we get $\operatorname{Lip}\left(u_{\infty}\right)>\lambda^{\star}=\operatorname{Lip}(\psi)$. This contradicts again the fact that $u_{\infty}$ is optimal for the limit problem.

Combining (3.2), (3.3), (3.4), and (3.8), together with the fact that $u_{\infty}$ and $\psi$ are $\infty$-harmonic in $\Omega^{*}$ with the same value on the boundary of this set, we end the proof of Theorem 2 .

\section{Proof of Theorem 3}

Now let us show that when $(\vec{H})$ does not hold there is no uniqueness for minimizers of the limit problem.

Proof of Theorem 3. As before, let $\lambda^{\star}$ be such that

$$
\Omega^{\star}:=\bigcup_{x \in \partial \Omega} B_{\frac{f(x)}{\lambda^{\star}}}(x) \cap \Omega
$$

has Lebesgue measure precisely $\alpha$, and assume that $(\underline{\mathrm{H}})$ does not hold. That is,

$$
\operatorname{Lip}(f)>\lambda^{\star} .
$$

Let

$$
D:=\bigcup_{x \in \partial \Omega} B_{\frac{f(x)}{\operatorname{Lip}(f)}}(x) \cap \Omega .
$$

We have

$$
\mathcal{L}^{n}(D)<\alpha
$$


By our previous result we have that

$$
\psi(x):=\max _{y \in \partial \Omega}(f(y)-\operatorname{Lip}(f)|x-y|)_{+}
$$

is an extremal for the limit problem with measure $\mathcal{L}^{n}(D)$.

Now, let $v_{\infty}$ be an extremal for the limit problem with measure $\alpha$. Then, as $v_{\infty}=f$ on $\partial \Omega$, we have

$$
\operatorname{Lip}\left(v_{\infty}\right) \geq \operatorname{Lip}(f)=\operatorname{Lip}(\psi) .
$$

On the other hand $\psi$ is a competitor in the limit problem with measure $\alpha$ and hence

We conclude that

$$
\operatorname{Lip}(\psi) \geq \operatorname{Lip}\left(v_{\infty}\right)
$$

$$
\operatorname{Lip}(\psi)=\operatorname{Lip}\left(v_{\infty}\right)=\operatorname{Lip}(f),
$$

and then $\psi$ is also a maximizer for the limit problem.

Moreover, we have that

$$
\psi(x) \leq v_{\infty}(x), \quad x \in D,
$$

if not the Lipschitz constant of $v_{\infty}$ is greater than $\operatorname{Lip}(\psi)$. Indeed, let us assume that there exists $x_{0} \in D$ such that

$$
\psi\left(x_{0}\right)>v_{\infty}\left(x_{0}\right) .
$$

That is,

$$
\max _{y \in \partial \Omega}\left(f(y)-\operatorname{Lip}(f)\left|x_{0}-y\right|\right)_{+}>v_{\infty}\left(x_{0}\right) .
$$

From that we get that there exists $y \in \partial \Omega$ such that

$$
f(y)-\operatorname{Lip}(f)\left|x_{0}-y\right|>v_{\infty}\left(x_{0}\right),
$$

which is to say that (using that $v_{\infty}=f$ on $\partial \Omega$ )

$$
v_{\infty}(y)-v_{\infty}\left(x_{0}\right)>\operatorname{Lip}(f)\left|x_{0}-y\right|,
$$

which clearly implies

$$
\operatorname{Lip}(f)<\operatorname{Lip}\left(v_{\infty}\right) .
$$

Therefore, we have that $\psi$ is the minimal extremal for the limit problem, and hence we obtain the following estimate for the support of any extremal $v_{\infty}$ :

$$
D:=\bigcup_{x \in \partial \Omega} B_{\frac{f(x)}{\operatorname{Lip}(f)}}(x) \cap \Omega \subset\left\{v_{\infty}>0\right\} .
$$

Now, let

$$
D_{\delta}:=\bigcup_{x \in \partial \Omega} B_{\frac{f(x)}{\operatorname{Lip}(f)}}(x) \cap \Omega+B(0, \delta),
$$

with $\delta$ small such that

$$
\mathcal{L}^{n}\left(D_{\delta}\right)<\alpha .
$$

In this set $D_{\delta}$, let us consider $v_{\infty}$ the solution to

$$
\left\{\begin{aligned}
\Delta_{\infty} v_{\infty}=0 & & \text { in } D_{\delta}, \\
v_{\infty}=f & & \text { on } \partial \Omega, \\
v_{\infty}=0 & & \text { on } \partial D_{\delta} .
\end{aligned}\right.
$$

Since $D \subset D_{\delta}$, we have

$$
\operatorname{Lip}\left(v_{\infty}\right)=\operatorname{Lip}(f) .
$$


To prove this fact, let us consider in the set $D_{\delta}$ the boundary value

$$
F(x)= \begin{cases}f(x) & x \in \partial \Omega, \\ 0 & x \in \partial D_{\delta} \cap \Omega .\end{cases}
$$

This boundary datum $F$ is a Lipschitz function with Lipschitz constant given by

$$
\operatorname{Lip}(F)=\sup _{x, y \in \partial D_{\delta}} \frac{|F(x)-F(y)|}{|x-y|} .
$$

Let us estimate this Lipschitz constant $\operatorname{Lip}(F)$. If $x, y \in \partial D_{\delta} \cap \Omega$, then

$$
\frac{|F(x)-F(y)|}{|x-y|}=0<\operatorname{Lip}(f) \text {. }
$$

When $x, y \in \partial \Omega$, clearly

$$
\frac{|F(x)-F(y)|}{|x-y|} \leq \operatorname{Lip}(f) .
$$

And finally when $x \in \partial \Omega$ and $y \in \partial D_{\delta} \cap \Omega$, we have

$$
\frac{|F(x)-F(y)|}{|x-y|}=\frac{\mid f(x)}{|x-y|}<\operatorname{Lip}(f) .
$$

We are using the fact that $D \subset \Omega_{\delta}$ and hence the distance $|x-y|$ is bigger than $f(x) / \operatorname{Lip}(f)$. To see this fact, just take $y \in \partial D$, then for any $x \in \partial \Omega$, we have

$$
f(x)-\operatorname{Lip}(f)|x-y| \leq 0,
$$

which is to say

$$
|x-y| \leq \frac{f(x)}{\operatorname{Lip}(f)}
$$

Therefore, we conclude that

$$
\operatorname{Lip}(F)=\operatorname{Lip}(f),
$$

and since $v_{\infty}$ has the same Lipschitz constant as $F$ (it is its best possible Lipschitz extension), we get that

$$
\operatorname{Lip}\left(v_{\infty}\right)=\operatorname{Lip}(f) .
$$

Hence $v_{\infty}$ is also an extremal for the limit problem that is positive on $\partial D \subset\left(D_{\delta}\right)^{o}$ (the strong maximum principle holds for $\infty$-harmonic functions), and hence we conclude that $v_{\infty} \neq \psi$.

With these estimates we can conclude that there is no strict monotonicity with respect to the measure in the limit problem.

Now, we can state further consequences of our previous results.

Theorem 7. Assume that

$$
\beta:=\mathcal{L}^{n}\left(\bigcup_{x \in \partial \Omega} B_{\frac{f(x)}{\operatorname{Lip}(f)}}(x) \cap \Omega\right)<\alpha .
$$

Then we have

$$
\lim _{p \rightarrow \infty} \mathfrak{P}_{p}(\alpha)=\lim _{p \rightarrow \infty} \mathfrak{P}_{p}(\beta)
$$

in the sense that if $u_{p}$ is an extremal for $\mathfrak{P}_{p}(\alpha)$ and $v_{p}$ is an extremal for $\mathfrak{P}_{p}(\beta)$, then

$$
\lim _{p \rightarrow \infty}\left(\int_{\Omega}\left|\nabla u_{p}(X)\right|^{p} d X\right)^{1 / p}=\lim _{p \rightarrow \infty}\left(\int_{\Omega}\left|\nabla v_{p}(X)\right|^{p} d X\right)^{1 / p} .
$$


Moreover,

uniformly in $\bar{\Omega}$ with

$$
v_{p} \rightarrow \psi \quad \text { and } \quad u_{p} \rightarrow u_{\infty}
$$

$$
\operatorname{Lip}\left(u_{\infty}\right)=\operatorname{Lip}(\psi)=\operatorname{Lip}(f) \quad \text { and } \quad \psi(x) \leq u_{\infty}(x) .
$$

One possible conclusion of this fact is that the boundary datum $f$ is so that the limit problem has many solutions and hence we are "wasting measure" when considering the problem with $\alpha$ instead of $\beta$. In fact, the value of the minimum for $\mathfrak{P}_{p}(\alpha)$ and for $\mathfrak{P}_{p}(\beta)$ are almost the same for $p$ large and the minimal solution of the limit problem is $\psi$ (which is the unique minimizer for $\mathfrak{P}_{\infty}(\beta)$ ).

\section{UNIFORM ESTIMATES AND FREE BOUNDARY CONVERGENCE ISSUES}

This section is devoted to establishing Theorems 4 , 5 and 6 To this end we shall revisit the study of the $p$-Dirichlet energy minimization problem with volume constraint, $\left[\mathfrak{P}_{p}\right.$ ) carried out in [21] and in [10]. Our strategy is to seize uniform-in- $p$ properties and afterwards explore their impact on the limiting problem $\left(\mathfrak{P}_{\infty}\right)$.

It is well established in the literature that ordinary techniques from the calculus of variations are not suitable to approach directly optimal design problems with volume constraints. Indeed, to establish existence of a minimizer for problem $\left(\mathfrak{P}_{p}\right.$ requires a careful analysis, involving penalty methods, and geometric measure perturbation techniques.

A penalized version of problem $\left(\mathfrak{P} p_{p}\right)$ can be easily set up. Indeed, for each $L>0$, let

$$
\varrho_{L}(t):=L(t-\alpha)^{+} .
$$

We then define the $L$-penalized problem for the $p$-Dirichlet integral as

$\left(\mathfrak{P}_{p}^{L}\right) \quad \min \left\{\int_{\Omega}|\nabla u(X)|^{p} d X+\varrho_{L}(\{u>0\}) \mid u \in W^{1, p}(\Omega), u=f\right.$ on $\left.\partial \Omega\right\}$.

Notice that problem $\left(\mathfrak{P}_{p}^{L}\right\rangle$ does not involve a volume constraint anymore, thus the proof of existence of a minimizer, $u_{p}^{L}$, for problem $\left(\mathfrak{P}_{p}^{L}\right)$ follows a standard scheme from the calculus of variations. It is also simple to check that $u_{p}^{L} \geq 0$ and $\Delta_{p} u_{p}^{L}$ is a nonnegative Radon measure supported on $\partial\left\{u_{p}^{L}>0\right\}$. In particular, $u_{p}^{L}$ is $p$-harmonic in its positivity set, that is, $u_{p}^{L}$ satisfies the following PDE:

$$
\Delta_{p} u_{p}^{L}=0, \quad \text { in }\left\{u_{p}^{L}>0\right\} .
$$

Although locally $C^{1, \alpha}$ within $\left\{u_{p}^{L}>0\right\}$, notice that Lipschitz is the optimal regularity for $u_{p}^{L}$ in $\Omega$. This is due to the fact that $\nabla u_{p}^{L}$ jumps from positive slope to zero along the free boundary $\partial\left\{u_{p}^{L}>0\right\}$. Indeed it has been proven in [21, 10, that for each $L$ fixed $u_{p}^{L}$ is locally Lipschitz continuous in $\Omega$. Our next lemma gives the precise dependence of the Lipschitz norm of $u_{p}^{L}$ with respect to $p$ and the penalty charge $L$. This lemma is essentially taken from [25]. We present a proof here as a courtesy to the readers.

Lemma 1. Let $u_{p}^{L}$ be a minimizer for $\left|\mathfrak{P}_{p}^{L}\right|$. Then,

$$
\left\|\nabla u_{p}^{L}\right\|_{L^{\infty}(\Omega)} \leq C L^{1 / p},
$$

where $C$ is a constant that depends only on dimension, $f$ and $\alpha$. 
Proof. Since we are interested in the limiting problem, we will only deal with the case $p \gg 1$. We will follow the approach suggested in [3], keeping track of the precise constants that appear on the estimates. From the minimality of $u_{p}^{L}$, we deduce, for any ball $B=B_{d}\left(x_{0}\right) \subset \Omega$ centered at a free boundary point, i.e., $x_{0} \in \partial\left\{u_{p}^{L}>0\right\}$, that there holds

$$
L \cdot \mathcal{L}^{n}\left(\left\{x \in B_{d}\left(x_{0}\right) \mid u_{p}^{L}(x)=0\right\}\right) \geq c_{0}\left(\int_{\Omega}\left|\nabla\left(u_{p}^{L}-\mathfrak{h}_{p}\right)(x)\right|^{p} d x\right),
$$

where $\mathfrak{h}_{p}$ is the $p$-harmonic function in $B_{d}\left(x_{0}\right)$ which agrees with $u_{p}^{L}$ on $\partial B_{d}\left(x_{0}\right)$ and $c_{0}$ is a constant that depends only upon dimension. For any direction $\nu$, we define

$$
r_{\nu}:=\min \left\{r \mid \frac{1}{4} \leq r \leq 1 \text { and } u_{p}^{L}\left(x_{0}+d r \nu\right)=0\right\}
$$

if such a set is nonempty; otherwise, we put $r_{\nu}=1$. Taking into account that

$$
u_{p}^{L}\left(x_{0}+d r_{\nu} \nu\right)=0
$$

whenever $r_{\nu}<1$, we can compute,

$$
\begin{aligned}
\mathfrak{h}_{p}\left(x_{0}+d r_{\nu} \nu\right) & =\int_{r_{\nu}}^{1} \frac{d}{d r}\left(u_{p}^{L}-\mathfrak{h}_{p}\right)\left(x_{0}+d r \nu\right) d r \\
& \leq d \cdot\left(1-r_{\nu}\right)^{1 / p^{\prime}} \times\left[\int_{r_{\nu}}^{1}\left|\nabla\left(\mathfrak{h}_{p}-u_{p}^{L}\right)\left(x_{0}+r \nu\right)\right|^{p} d r\right]^{1 / p} .
\end{aligned}
$$

Here $\frac{1}{p}+\frac{1}{p^{\prime}}=1$. Now, by the Harnack inequality, we know that

$$
\inf _{B_{\frac{2}{3} d}\left(x_{0}\right)} \mathfrak{h}_{p} \geq c_{1} \mathfrak{h}_{p}\left(x_{0}\right)
$$

for a constant $c_{1}>0$ that depends only on dimension (see, for instance, 15]). Let us consider the following barrier function, $b$, given by

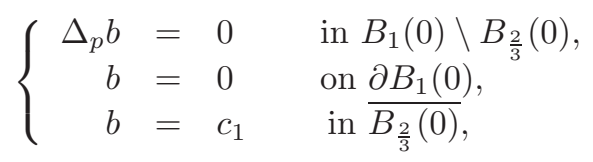

where $c_{1}$ is the universal constant in (5.4). By the Hopf maximum principle, there exists a universal constant $c_{2}>0$, depending only on dimension, such that

$$
b(x) \geq c_{2}(1-|x|) .
$$

By the maximum principle and (5.6) we can write

$$
\mathfrak{h}_{p}\left(x_{0}+d x\right) \geq \mathfrak{h}_{p}\left(x_{0}\right) \cdot b(x) \geq c_{2} \mathfrak{h}_{p}\left(x_{0}\right) \cdot(1-|x|) .
$$

Combining (5.2) and (5.7), we end up with

$$
d^{p} \cdot\left[\int_{r_{\nu}}^{1}\left|\nabla\left(\mathfrak{h}_{p}-u_{p}^{L}\right)\left(x_{0}+r \nu\right)\right|^{p} d r\right] \geq c_{3} \mathfrak{h}_{p}^{p}\left(x_{0}\right) \cdot\left(1-r_{\nu}\right) .
$$

Integrating (5.8) with respect to $\nu$ over $\mathbb{S}^{n-1}$ and taking into account the definition of $r_{\nu}$, we find

$$
\left(\frac{\mathfrak{h}_{p}(x)}{d}\right)^{p} \cdot \int_{B_{d}(x) \backslash B_{d / 4}(x)} \chi_{\left\{u_{p}^{L}=0\right\}} d x \leq C_{4} \int_{B_{d}(x)}\left|\nabla\left(\mathfrak{h}_{p}-u_{p}^{L}\right)(x)\right|^{p} d x .
$$


If we replace, in all of our arguments so far, $B_{d / 4}(x)$ by $B_{d / 4}(\bar{x})$, for any $\bar{x} \in$ $\partial B_{d / 2}(x)$, we obtain

$$
\left(\frac{\mathfrak{h}_{p}(x)}{d}\right)^{p} \cdot \int_{B_{d}(x) \backslash B_{d / 4}(\bar{x})} \chi_{\left\{u_{p}^{L}=0\right\}} d x \leq \tilde{C}_{4} \int_{B_{d}(x)}\left|\nabla\left(\mathfrak{h}_{p}-u_{p}^{L}\right)(x)\right|^{p} d x
$$

for every $\bar{x} \in \partial B_{d / 2}(x)$.

Integrating (5.10) with respect to $\bar{x}$, yields

$$
\left(\frac{\mathfrak{h}_{p}(x)}{d}\right)^{p} \cdot\left|\left\{x \in B_{d}(x) \mid u_{p}^{L}(x)=0\right\}\right| \leq C_{5} \int_{B_{d}(x)}\left|\nabla\left(\mathfrak{h}_{p}-u_{p}^{L}\right)(x)\right|^{p} d x
$$

Now we argue as follows: let $\rho:=\operatorname{dist}\left(x, \partial\left\{u_{p}^{L}>0\right\}\right)$ and for each $0<\delta \ll 1$, denote $\mathfrak{h}_{p}^{\delta}$ the $p$-harmonic function in $B_{\rho+\delta}(x)$ that agrees with $u_{p}^{L}$ on $\partial B_{\rho+\delta}(x)$. Combining (5.2) and (5.11) together with standard elliptic estimate, we deduce

$$
u_{p}^{L}(x)=\mathfrak{h}_{\delta}(x)+\mathrm{o}(1) \leq C_{6} L^{1 / p}(\rho+\delta)+\mathrm{o}(1), \quad \text { as } \delta \searrow 0,
$$

for a constant $C_{6}$ that depends on dimension, $f$ and $\alpha$. Letting $\delta \searrow 0$ in (5.12) we finally conclude

$$
u_{p}^{L}(x) \leq C_{6} L^{1 / p} \operatorname{dist}\left(x, \partial \Omega_{\lambda}^{\star}\right),
$$

which clearly implies that $u_{p}^{L}$ is Lipschitz continuous up to the free boundary $\partial\left\{u_{p}^{L}>0\right\}$ and $\left\|\nabla u_{p}^{L}\right\|_{\infty} \lesssim L^{1 / p}$. Lemma 1 is proved.

Another important piece of information concerns uniform nondegeneracy.

Lemma 2. Let $x \in\left\{u_{p}^{L}>0\right\}$ be a free boundary point. Then

$$
L^{-1 / p} \underline{c} \cdot \operatorname{dist}\left(x, \partial\left\{u_{p}^{L}>0\right\}\right) \leq u_{p}^{L}(x)
$$

for a constant $\underline{c}$ that depends only upon dimension, $f$ and $\alpha$. Moreover, the following strong nondegeneracy holds

$$
\sup _{B_{r}\left(x_{0}\right)} u_{p}^{L} \geq L^{-1 / p} \underline{c}_{1} r
$$

for any free boundary point $x_{0} \in \partial\left\{u_{p}^{L}>0\right\}$. The constant $\underline{c}_{1}$ depends only on dimension, $f$ and $\alpha$ and is independent of $p$.

The proof of Lemma 2 is, by now, classical in variational free boundary theory. It relies on "cutting" a small hole around the free boundary point and comparing the result with the original optimal design. For further details we refer the readers to 25. Theorem 6.2. As observed in the proof of Lemma 1, the fact that $\underline{c}$ and $\underline{c}_{1}$ are universal is a consequence of a uniform-in- $p$ Harnack inequality and a uniform-in- $p$ Hopf boundary maximum principle. We skip the details here.

The penalty method strategy is based on the idea that if $L$ is large enough (but still finite), one expects that minimizers for $\left(\mathfrak{P}_{p}^{L}\right)$ would rather prefer to obey the volume constraint, $\mathcal{L}^{n}\left(\left\{u_{p}^{L}>0\right\}\right) \leq \alpha$. Therefore, they will be a solution for the original problem, $\left(\mathfrak{\mathfrak { P } _ { p }}\right)$. Such a strategy does work, 21], 10], and 25], however it relies on a fine geometric measure perturbation approach. The following theorem is a consequence of the analysis carried out in [25, section 7].

Lemma 3. There exists a universal constant $C$, depending only on dimension, $f$ and $\alpha$, but independent of $p$, such that if

$$
L \geq C p,
$$


then

$$
\mathcal{L}^{n}\left(\left\{u_{p}^{L}>0\right\}\right) \leq \alpha .
$$

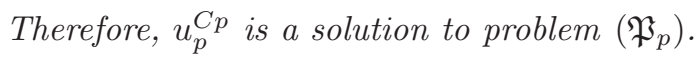

It is important to notice that any minimizer, $u_{p}$, of problem $\left(\mathfrak{P}_{p}\right)$ is also a minimizing function for problem $\left(\mathfrak{P}_{p}^{C p}\right)$. As a consequence, combining Lemmas 1 , 2. and 3, we obtain the following theorem with estimates that are uniform in $p$.

Theorem 8. There exists a constant $K>0$, depending on dimension, $f$ and $\alpha$, but independent of $p$, such that for any solution $u_{p}$ of $\left(\mathfrak{P}_{p}\right)$, there holds

$$
\left\|\nabla u_{p}\right\|_{L^{\infty}(\Omega)} \leq K \text {. }
$$

Moreover, $u_{p}$ grows linearly uniform-in-p away from the free boundary; that is, for a constant $\gamma>0$ independent of $p$,

$$
u_{p}(x) \geq \gamma \operatorname{dist}\left(x, \partial\left\{u_{p}>0\right\}\right), \quad \forall x \in\left\{u_{p}>0\right\} .
$$

In addition, $u_{p}$ is uniformly strong nondegenerate; that is, for any fixed free boundary point $x_{0} \in \partial\left\{u_{p}>0\right\}$,

$$
\sup _{B_{r}\left(x_{0}\right)} u_{p} \geq \gamma r
$$

where $\gamma>0$ is independent of $p$.

Proof of Theorem 4. Notice that $\lim _{p \rightarrow \infty} p^{1 / p}=1$. Passing the limit as $p$ goes to infinity in (5.15), (5.16), and (5.17), we prove Theorem 4 .

Theorem 8 actually gives more qualitative information than Theorem 4 itself. Indeed, with Theorem 8 we can address free boundary convergence issues. In what follows we prove convergence of the free boundaries in the Hausdorff metric, Theorem 5 .

Proof of Theorem 5. For any set $A \subset \mathbb{R}^{n}$, and $\varepsilon>0$ fixed, let $\Gamma_{\varepsilon}(A)$ denote the $\varepsilon$-neighborhood of $A$; that is,

$$
\Gamma_{\varepsilon}(A):=\left\{x \in \mathbb{R}^{n} \mid \operatorname{dist}(x, A)<\varepsilon\right\} .
$$

We have to show that given $\varepsilon>0$, for $p \gg 1$, depending on $\varepsilon>0$, there hold

$$
\partial\left\{u_{p}>0\right\} \subset \Gamma_{\varepsilon}\left(\partial\left\{u_{\infty}>0\right\}\right)
$$

and

$$
\partial\left\{u_{\infty}>0\right\} \subset \Gamma_{\varepsilon}\left(\partial\left\{u_{p}>0\right\}\right)
$$

Let $\xi$ be an arbitrary point on $\partial\left\{u_{p}>0\right\}$, and let us assume, for sake of contradiction, that $\xi \notin \Gamma_{\varepsilon}\left(\partial\left\{u_{\infty}>0\right\}\right)$; that is,

$$
\operatorname{dist}\left(\xi, \partial\left\{u_{\infty}>0\right\}\right) \geq \varepsilon .
$$

If $u_{\infty}(\xi)>0$, then by linear growth we would have

$$
u_{\infty}(\xi) \geq \gamma \operatorname{dist}\left(\xi, \partial\left\{u_{\infty}>0\right\}\right) \geq \gamma \varepsilon
$$

Thus, from uniform convergence, if $p \gg 1, u_{p}(\xi) \geq \frac{2}{3} \gamma \varepsilon$, driving us to a contradiction. If we assume $u_{\infty}(\xi)=0$, then $\left.u_{\infty}\right|_{B_{\varepsilon}(\xi)} \equiv 0$. However, by strong nondegeneracy, we know that

$$
\sup _{B \frac{\varepsilon}{2}} u_{p} \geq \gamma_{0} \frac{\varepsilon}{2},
$$


and again it would drive us to a contradiction on the uniform convergence of $u_{p}$ to $u_{\infty}$. We have proven

$$
\partial\left\{u_{p}>0\right\} \subset \Gamma_{\varepsilon}\left(\partial\left\{u_{\infty}>0\right\}\right) .
$$

The other inclusion is proven similarly.

Proof of Theorem 6. Initially, let us recall some further facts from the $p$-Dirichlet minimization problem $\left(\overline{\mathfrak{P}_{p}}\right)$. Recall that the free boundary $\partial\left\{u_{p}>0\right\}$ is a $C^{1, \alpha}$ smooth surface up to an $\mathcal{H}^{n-1}$ closed and negligible set (see [10], 21], 8] ). From the free boundary condition $\left|\nabla u_{p}\right|=\lambda_{p}$, we deduce that

$$
\lim _{\substack{x \rightarrow \partial \Omega_{p} \\ x \in \Omega_{p}}} \frac{u_{p}(x)}{\operatorname{dist}\left(x, \partial \Omega_{p}\right)}=\lambda_{p} .
$$

Hereafter, $\Omega_{p}$ denotes the set of positivity of $u_{p}$. From uniform convergence, $u_{p} \rightrightarrows$ $u_{\infty}$, given a point $x \in \Omega_{\infty}$, we may assume $x \in \Omega_{p}$ for $p$ sufficiently large. Now, from the free boundary convergence result, Theorem 5 , there holds

$$
\operatorname{dist}\left(x, \partial \Omega_{\infty}\right)=\operatorname{dist}\left(x, \partial \Omega_{p}\right)+o(1), \quad \text { as } p \nearrow \infty .
$$

Here, $o(1)$ is an error that goes to zero as $p$ goes to infinity. Thus, using once more the Hausdorff metric convergence of the free boundary and uniform convergence of $u_{p}$ to $u_{\infty}$ together with (5.18) and (5.19), we reach the chain

$$
\begin{aligned}
\frac{u_{\infty}(x)}{\operatorname{dist}\left(x, \partial \Omega_{\infty}\right)} & =\frac{u_{p}(x)}{\operatorname{dist}\left(x, \partial \Omega_{p}\right)}+o(1) \\
& =\lambda_{p}+o\left(\operatorname{dist}\left(x, \partial \Omega_{p}\right)\right)+o(1) \\
& =\lambda_{\infty}+o\left(\operatorname{dist}\left(x, \partial \Omega_{\infty}\right)\right)+o(1) .
\end{aligned}
$$

Letting $p \rightarrow \infty$, the proof of Theorem 6 is complete.

\section{ACKNOWLEDGMENTS}

The first author was partially supported by MTM2004-02223, MEC, Spain, by UBA X066 and by CONICET, Argentina. The second author's research was partially supported by CNPq-Brazil.

\section{REFERENCES}

1. A. Acker and R. Meyer. A free boundary problem for the p-Laplacian: uniqueness, convexity, and successive approximation of solutions. Electron. J. Differential Equations 1995, No. 08, 20 pp. (electronic). MR.1334863(96c:35198)

2. N. Aguilera, H. Alt and L. Caffarelli, An optimization problem with volume constraint, SIAM J. Control Optim. 24, (1986), 191-198. MR826512 (87d:49010)

3. H. Alt and L. Caffarelli, Existence and regularity for a minimum problem with regularity, J. Reine Angew. Math. 325, (1981), 105-144. MR618549 (83a:49011)

4. N. Aguilera, L. Caffarelli and J. Spruck, An optimization problem in heat conduction, Ann. Scuola Norm. Sup. Pisa Cl. Sci. (4) 14 (1987), 355-387. MR951225 (89h:49016)

5. G. Aronsson, M. G. Crandall and P. Juutinen, A tour of the theory of absolutely minimizing functions. Bull. Amer. Math. Soc., 41 (2004), 439-505. MR 2083637 (2005k:35159)

6. T. Bhattacharya, E. Di Benedetto and J. J. Manfredi, Limits as $p \rightarrow \infty$ of $\Delta_{p} u_{p}=f$ and related extremal problems. Rend. Sem. Mat. Univ. Politec. Torino, (1991), 15-68. MR 1155453 (93a:35049)

7. M. G. Crandall, H. Ishii and P. L. Lions. User's guide to viscosity solutions of second order partial differential equations. Bull. Amer. Math. Soc., 27 (1992), 1-67. MR:1118699|(92j:35050)

8. D. Danielli and A. Petrosyan, A minimum problem with free boundary for a degenerate quasilinear operator. Calc. Var. Partial Differential Equations 23 (2005), no. 1, 97-124. MR2133664 (2006c:35303) 
9. L. C. Evans and W. Gangbo, Differential equations methods for the Monge-Kantorovich mass transfer problem. Mem. Amer. Math. Soc., 137 (1999), no. 653. MR1464149 (99g:35132)

10. J. Fernández Bonder, S. Martínez, and N. Wolanski, An optimization problem with volume constraint for a degenerate quasilinear operator. J. Differential Equations 227 (2006), 80-101. MR 2233955 (2007b:49007)

11. J. García-Azorero, J. J. Manfredi, I. Peral and J. D. Rossi, The Neumann problem for the $\infty$-Laplacian and the Monge-Kantorovich mass transfer problem. Nonlinear Analysis TM\&A., 66, (2007), 349-366. MR2279530 (2008f:35148)

12. A. Henrot and H. Shahgholian, Convexity of free boundaries with Bernoulli type boundary condition. Nonlinear Anal. 28 (1997), 815-823. MR:1422187 (97j:35165)

13. A. Henrot and H. Shahgholian, Existence of classical solutions to a free boundary problem for the p-Laplace operator. I. The exterior convex case. J. Reine Angew. Math. 521 (2000), 85-97. MR 1752296 (2001f:35442)

14. A. Henrot and H. Shahgholian, Existence of classical solutions to a free boundary problem for the p-Laplace operator. II. The interior convex case. Indiana Univ. Math. J. 49 (2000), 311-323. MR:1777029(2001m:35326)

15. P. Koskela, J. J. Manfredi, and E. Villamor, Regularity theory and traces of $\mathcal{A}$-harmonic functions. Trans. Amer. Math. Soc. 348 (1996), 755-766. MR1311911 (96g:35063)

16. J. L. Lewis and A.L. Vogel, Uniqueness in a free boundary problem. Comm. Partial Differential Equations 31 (2006), 1591-1614. MR2273966 (2007h:35373)

17. C. Lederman, A free boundary problem with a volume penalization. Ann. Scuola Norm. Sup. Pisa Cl. Sci. (4) 23 (1996), 249-300. MR1433424 (98a:35145)

18. J. J. Manfredi, A. Petrosyan and H. Shahgholian, A free boundary problem for $\infty$ Laplace equation. Calc. Var. Partial Differential Equations 14 (2002), 359-384. MR 1899452 (2003a:35212)

19. S. Martinez, An optimization problem with volume constrain in Orlicz spaces. J. Math. Anal. Appl., 340 (2008), 1407-1421. MR2390940 (2010b:49003)

20. S. Martinez and N. Wolanski, A minimum problem with free boundary in Orlicz spaces, Adv. Mathematics 218 (2008), 1914-1971. MR2431665 (2009h:35456)

21. K. Oliveira and E. V. Teixeira, An optimization problem with free boundary governed by a degenerate quasilinear operator. Differential Integral Equations 19 (2006), 1061-1080. MR2262097(2007h:35374)

22. E. V. Teixeira, The nonlinear optimization problem in heat conduction. Calc. Var. Partial Differential Equations 24 (2005), 21-46. MR2157849(2006d:49005)

23. E. V. Teixeira, Uniqueness, symmetry and full regularity of free boundary in optimization problems with volume constraint. Interfaces and Free Boundaries 9 (2007), 133-148. MR 2317302 (2007m:49002)

24. E. V. Teixeira, A variational treatment for elliptic equations of the flame propagation type: regularity of the free boundary. Ann. Inst. H. Poincaré Anal. Non Linéaire 25 (2008), 633-658. MR2436786 (2010a:35284)

25. E. V. Teixeira, Optimal design problems in rough inhomogeneous media. Existence theory. Preprint. arXiv:0710.2936.

26. E. V. Teixeira, Optimal design problems in rough inhomogeneous media. Free boundary regularity theory. In preparation.

Departamento de Análisis Matemático, Universidad de Alicante, Alicante, Spain

E-mail address: jrossi@dm.uba.ar

Departamento de Matemática, Universidade Federal do Ceará, Campus do Pici Bloco 914, Fortaleza, CE - Brazil 60.455-760

E-mail address: eteixeira@ufc.br 